15,09

\title{
Ориентационный порядок в жидкокристаллических комплексах на основе лантаноидов
}

\author{
(С) Л.А. Добрун, А.П. Ковшик, Е.И. Рюмцев, А.А. Калинкин \\ Санкт-Петербургский государственный университет, \\ Санкт-Петербург, Россия \\ E-mail: I.dobrun@spbu.ru
}

(Поступила в Редакцию 7 июля 2017 г.

В окончательной редакции 28 сентября 2017 г.)

\begin{abstract}
Впервые для серии жидкокристаллических комплексов на основе лантаноидов $\left(\mathrm{Eu}^{+3}, \mathrm{Gd}^{+3}, \mathrm{~Tb}^{+3}, \mathrm{Dy}^{+3}\right) \mathrm{c}$ одинаковым лигандным составом в температурном интервале существования нематической фазы определена степень ориентационного порядка $S$ с использованием экспериментальных результатов рефрактометрии. Обнаружена четно-нечетная альтернация $S$ при последовательном увеличении количества протонов в ионах комплексообразователя. Полученные значения $S$ сопоставляются с соответствующими величинами степени порядка каламитных органических жидких кристаллов.
\end{abstract}

Работа выполнена при финансовой поддержке СПбГУ (гранты № 11.41.455.2017 и 11.41.435.2017).

DOI: 10.21883/FTT.2018.04.45697.227

Жидкокристаллические координационные соединения на основе лантаноидов (лантанидомезогены) обладают уникальным сочетанием магнитных, люминесцентных и жидкокристаллических свойств. Надмолекулярная организация лантанидомезогенов позволяет получать высокоэффективные люминесцентные среды для использования, например, в качестве компонентов различных органических оптоэлектронных устройств. Поэтому в последние годы интенсивно изучаются физические свойства лантанидомезогенов и возможности их практического применения. Для целенаправленного синтеза лантанидомезогенов с заданными свойствами необходимо установить связь между химической структурой комплексов и их физическими свойствами. Результаты исследования магнитных и свойств лантанидомезогенов показали, что замена иона лантаноида в комплексах с одинаковым лигандным составом может изменять величину анизотропии магнитной восприимчивости на порядок и даже изменять ее знак [1]. Для установления влияния иона комплексообразователя на оптическую анизотропию $\Delta n=n_{e}-n_{o}\left(n_{e}\right.$ и $n_{o}$ - главные показатели преломления) лантанидомезогенов были определены $n_{e}$ и $n_{o}$ ряда комплексов на основе лантаноидов с последовательным изменением зарядового числа $Z$ и одинаковым лигандным окружением [2]. В качестве объектов исследования были использованы жидкокристаллические комплексы лантаноидов $\operatorname{Ln}\left(\mathrm{CPDk}_{3-5}\right) \mathrm{Bpy}_{17-17}$, где $\mathrm{Ln}-$ ион-комплексообразователь $\left(\mathrm{Eu}^{+3}, \mathrm{Gd}^{+3}, \mathrm{~Tb}^{+3}, \mathrm{Dy}^{+3}\right)$; $\mathrm{CPDk}_{3-5}$ - 1-[4-(4-пропилциклогексил)фенил]октан-1,3дион, Вру $17-17-5,5^{\prime}$-ди(гептадецил)-2,2'-бипиридин. Величины оптической анизотропии большинства органических каламитных жидких кристаллов в несколько раз превышают $\Delta n$ исследованных комплексов. При сопоставлении величин $\Delta n$ исследованных комплексов при одинаковой относительной температуре $T_{o}-T\left(T_{o}\right.$ - температура перехода жидкий кристалл- изотропная жидкость) обнаружен четно-нечетный эффект в зависимости оптической анизотропии от количества протонов в ионах комплексообразователя. Величина $\Delta n$ изменялась в среднем на $17 \%$. Вариация оптической анизотропии может быть вызвана, как изменением анизотропии электронной поляризуемости комплексов $\Delta b=b_{1}-b_{2}\left(b_{1}\right.$ и $b_{2}-$ поляризуемости вдоль длинной и поперечной молекулярных осей соответственно), так и изменением в мезофазе степени ориентационного порядка $S$. Вопрос о степени влияния $S$ и $\Delta b$ на величину оптической анизотропии жидкого кристалла может быть решен путем экспериментального определения этих величин.

Целью настоящей работы было определение величин $S$ в температурном интервале существования нематической фазы и установление влияния комплексообразователя на ориентационную упорядоченность комплексов $\operatorname{Ln}\left(\mathrm{CPDk}_{3-5}\right) \mathrm{Bpy}_{17-17}$.

Степень ориентационного порядка жидких кристаллов связана с молярными рефракциями $R_{e}, R_{o}, R_{i s}$ и анизотропией рефракции $\Delta R=R_{e}-R_{o}$ соотношениями (1) $-(5)$ :

$$
\begin{gathered}
R_{e}=\left(\frac{n_{e}^{2}-1}{\left\langle n^{2}\right\rangle+2}\right) \frac{M}{\rho}=\frac{4}{3} \pi N_{A}\left(b+\frac{2}{3} \Delta b S\right), \\
R_{o}=\left(\frac{n_{o}^{2}-1}{\left\langle n^{2}\right\rangle+2}\right) \frac{M}{\rho}=\frac{4}{3} \pi N_{A}\left(b-\frac{1}{3} \Delta b S\right), \\
\Delta R=R_{o}-R_{e}=\left(\frac{n_{e}^{2}-1}{\left\langle n^{2}\right\rangle+2}-\frac{n_{o}^{2}-1}{\left\langle n^{2}\right\rangle+2}\right) \frac{M}{\rho} \\
=\frac{4}{3} \pi N_{A} \Delta b S, \\
R_{i s}=\left(\frac{n_{i s}^{2}-1}{\left\langle n_{i s}^{2}\right\rangle+2}\right) \frac{M}{\rho}=\frac{4}{3} \pi N_{A} b,
\end{gathered}
$$




$$
\frac{\Delta R}{R_{i s}}=\frac{\Delta b}{b} S .
$$

Здесь $b-$ средняя поляризуемость молекулы, $\Delta b-$ анизотропия поляризуемости, $M-$ молекулярная масса, $\rho-$ плотность, $N_{A}-$ число Авогадро, $\left\langle n^{2}\right\rangle=\left(n_{e}^{2}+2 n_{o}^{2}\right) / 3$ - среднее значение квадрата показателя преломления. Знаменателем в формулах является $\left\langle n^{2}\right\rangle+2$, так как при малой величине $\Delta n$ рассматриваемых лантанидомезогенов действующее на молекулу электрическое поле можно считать изотропным.

Для определения $S$ жидких кристаллов можно использовать экспериментально полученные значения $n_{e}$ и $n_{o}$, соотношение (3) и экстраполяцию линейной зависимости $\lg (\Delta R)$ от $\lg \left(T_{o}-T\right)$ или $\lg \left(1-T / T_{o}\right)$ к температуpe $0 \mathrm{~K}[3-5]$. В предположении, что при $T=0 \mathrm{~K}$ степень ориентационного порядка жидких кристаллов равна единице, определяются величины $\Delta b$ и, следовательно, значения $S$ в жидкокристаллической фазе.

При отсутствии экспериментальных данных о плотности $\rho$ жидкого кристалла, необходимых для вычисления $\Delta R$, экстраполяция зависимости $\lg \left(\Delta R / R_{i s}\right)$ от $\lg \left(T_{o}-T\right)$ к температуре $0 \mathrm{~K}$ позволяет в соответствии с уравнением (5) получить отношение анизотропии поляризуемости молекул к средней поляризуемости $\Delta b / b$ и, следовательно, найти степень ориентационного порядка $S$.

В настоящей работе с использованием температурных зависимостей главных показателей преломления лантанидомезогенов $n_{e}, n_{o}$ и $n_{i s}$ [2] были вычислены отношения анизотропии рефракции к рефракции в изотропной фазе $\Delta R / R_{i s}=\left(n_{e}^{2}-n_{o}^{2}\right) /\left(\left\langle n^{2}\right\rangle-1\right)$ в интервале температур существования нематической фазы. На рис. 1 представлена экстраполяция зависимостей $\lg \left(\Delta R / R_{i s}\right)$ от $\lg \left(T_{o}-T\right)$ к температуре $0 \mathrm{~K}$ для комплексов европия, гадолиния, тербия и диспрозия. Проходящие через экспериментальные точки линии должны пересекать нормали к оси $\lg \left(T_{o}-T\right)$ в точках $\lg \left(T_{o}-0\right)$. Поскольку температуры перехода в изотропную фазу $T_{o}$ для исследованных лантанидомезогенов близки (см. таблицу), нормали для различных лантанидомезогенов представлены на обсуждаемом рисунке единой линией.

С использованием значений $\lg \left(\Delta R / R_{i s}\right)$, полученных при пересечении экстраполяционных прямых с нормалью, были определены величины $\Delta b / b$ (см. таблицу),

Сокращенные обозначения исследованных лантанидомезогенов, количество протонов в ядре иона-комплексообразователя $Z$, температуры фазовых переходов $T_{o}$, отношение анизотпропии поляризуемости комплексов к средней поляризуемости $\Delta b / b$

\begin{tabular}{l|c|c|c}
\hline Лантанидомезогены & $Z$ & $T_{o},{ }^{\circ} \mathrm{C}$ & $\Delta b / b$ \\
\hline $\mathrm{Eu}\left(\mathrm{CPDk}_{3-5}\right)_{3} \mathrm{Bpy}_{17-17}$ & 63 & 131 & 0.19 \\
$\mathrm{Gd}\left(\mathrm{CPDk}_{3-5}\right)_{3} \mathrm{Bpy}_{17-17}$ & 64 & 137 & 0.21 \\
$\mathrm{~Tb}\left(\mathrm{CPDk}_{3-5}\right)_{3} \mathrm{Bpy}_{17-17}$ & 65 & 137 & 0.22 \\
$\mathrm{Dy}\left(\mathrm{CPDk}_{3-5}\right)_{3} \mathrm{Bpy}_{17-17}$ & 66 & 160 & 0.22
\end{tabular}

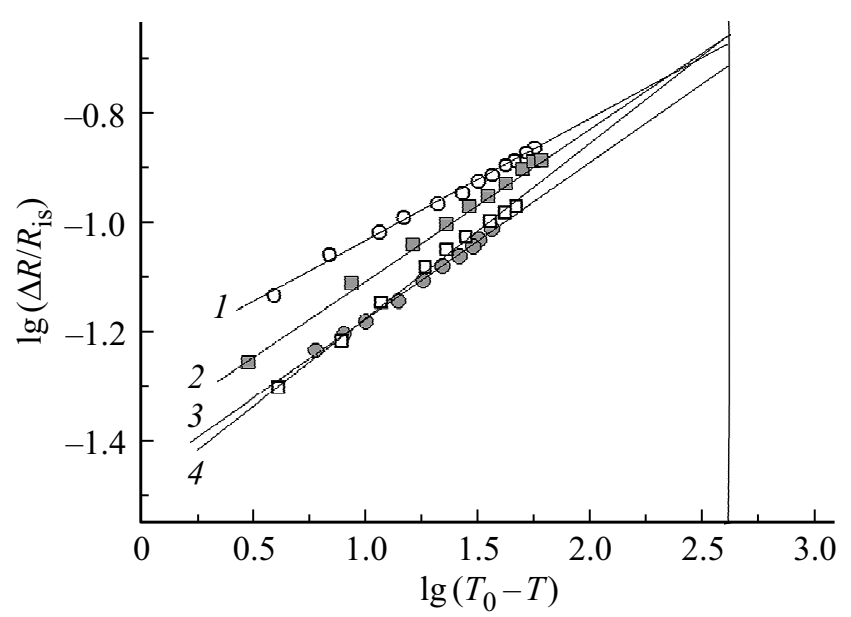

Рис. 1. Зависимости $\lg \left(\Delta R / R_{i s}\right)$ от относительной температуры $\lg \left(T_{o}-T\right)$ для лантанидомезогенов: $1-\mathrm{Gd}\left(\mathrm{CPDk}_{3-5}\right) \mathrm{Bpy}_{17-17}, 2-\mathrm{Dy}\left(\mathrm{CPDk}_{3-5}\right) \mathrm{Bpy}_{17-17}$, $3-\mathrm{Eu}\left(\mathrm{CPDk}_{3-5}\right) \mathrm{Bpy}_{17-17}, 4-\mathrm{Tb}\left(\mathrm{CPDk}_{3-5}\right) \mathrm{Bpy}_{17-17}$.

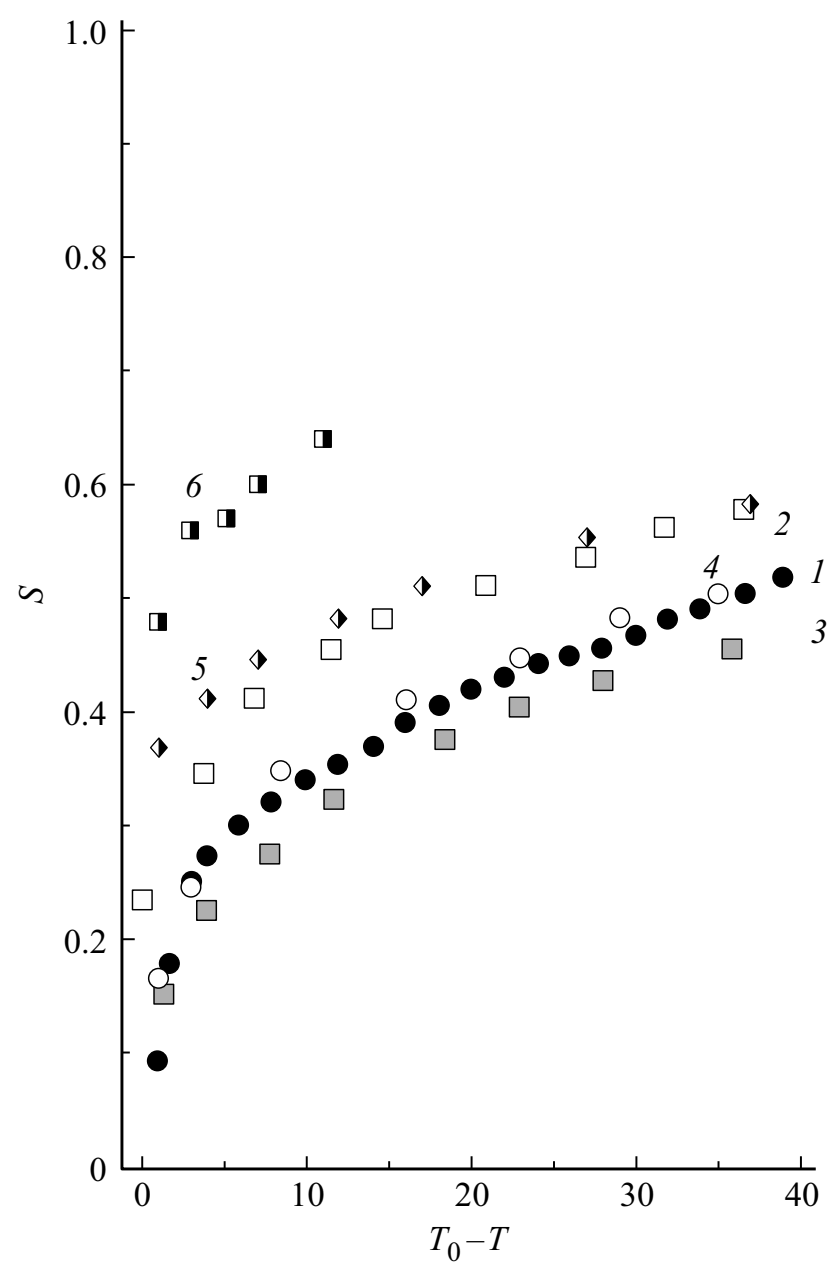

Рис. 2. Зависимость степени ориентационного порядка $S$ от относительной температуры $T_{o}-T$ : $1-\mathrm{Eu}\left(\mathrm{CPDk}_{3-5}\right) \mathrm{Bpy}_{17-17}, 2-\mathrm{Gd}\left(\mathrm{CPDk}_{3-5}\right) \mathrm{Bpy}_{17-17}$, $3-\mathrm{Tb}\left(\mathrm{CPDk}_{3-5}\right) \mathrm{Bpy}_{17-17}, 4-\mathrm{Dy}\left(\mathrm{CPDk}_{3-5}\right) \mathrm{Bpy}_{17-17}$, 5 - ПАА, $6-5 Ц$ Ц. 


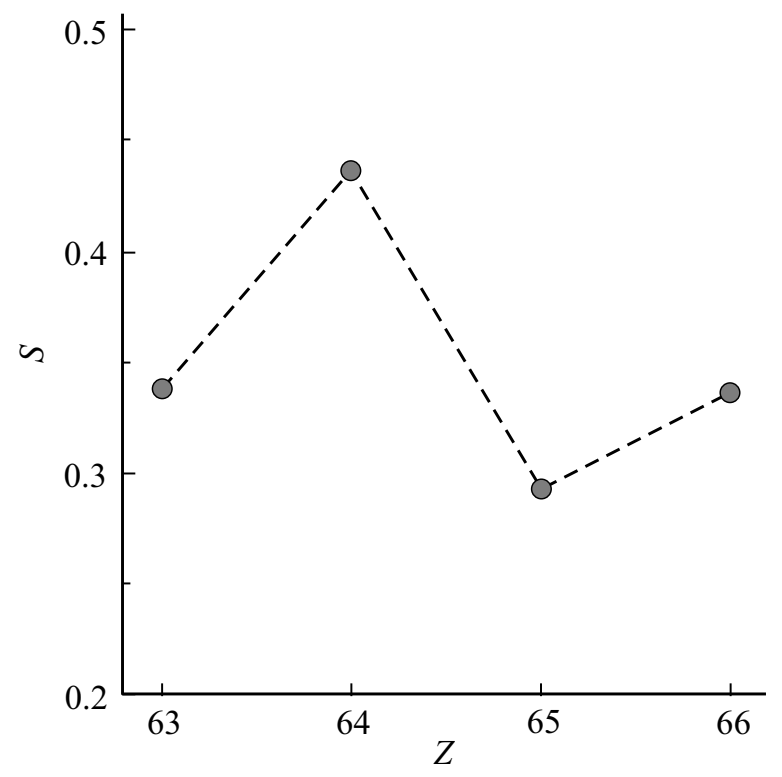

Рис. 3. Зависимость степени ориентационного порядка $S$ от количества протонов $Z$ в ионе комплексообразователя.

которые для исследованных лантанидомезогенов практически совпадают. Это возможно в случае постоянства величин $\Delta b$ и $b$ при вариации комплексообразователя или при пропорциональном изменении $\Delta b$ и $b$. Для всех исследованных образцов из отношения значений $\Delta R / R_{i s}$ к $\Delta b / b$ были получены зависимости $S$ от относительной температуры $T_{o}-T$ (рис. 2). Там же для сравнения показаны температурные зависимости $S$ органических жидких кристаллов пентилцианобифенила (5ЦБ) и nара-азоксианизола (ПАА) [6]. Из рисунка видно, что ориентационная упорядоченность 5ЦБ и ПАА несколько превосходит ориентационную упорядоченность исследованных лантанидомезогенов, а также что смена ионакомплексообразователя изменяет величину $S$ исследованных лантанидомезогенов. Для более наглядного представления влияния вариации иона комплексообразователя на ориентационную упорядоченность лантанидомезогенов на рис. 3 показана зависимость $S$ от количества протонов $Z$ в ионах комплексообразователей $\mathrm{Eu}, \mathrm{Gd}$, $\mathrm{Tb}$, Dу при относительной температуре $T_{o}-T=10^{\circ} \mathrm{C}$. Рис. 3 демонстрирует четно-нечетную альтернацию $S$ при последовательном увеличении $Z$ в ряду исследованных лантанидомезогенов. При этом вариация комплексообразователя изменяет $S$ комплексов в среднем на $12 \%$. Величины $S$ для лантанидомезогенов на основе ионов с четным $Z$ превышают ориентационную упорядоченность комплексов с нечетным количеством протонов.

Таким образом, впервые определена фундаментальная термодинамическая характеристика жидких кристаллов - степень ориентационного порядка мезофазы $S$ жидкокристаллических координационных соединений на основе лантаноидов. Дальнейшие исследования оптических свойств жидкокристаллических комплексов должны быть направлены на расширение круга изучаемых лантанидомезогенов путем вариации комплексообразователя и лигандного окружения.

\section{Список литературы}

[1] V.I. Dzhabarov, A.A. Knyazev, M.V. Strelkov, E.Yu. Molostova, V.A. Schustov, W. Haase, Yu.G. Galyametdinov. Liquid Crystals 37, 285 (2010).

[2] Л.А. Добрун, А.П. Ковшик, Е.И. Рюмцев, А.А. Князев, Ю.Г. Галяметдинов. ФТТ 59, 797(2017).

[3] H.A. Haller, H.R. Huggins, T.R. Lilienthal, Mc. Guire. J. Phys. Chem. 77, 950 (1973).

[4] Sushmita Sen, Pradir Brahma, Subir. K. Roy, D.K. Mukherjee, S.B. Roy. Mol. Cryst. Liq. Cryst. 100, 327 (1983).

[6] S. Chandrasekhar, N.V. Madhusudana. J. de Phys. Coll. C4. 30, 4 (1969). 